\title{
EVALUATION OF ARTHROCENTESIS WITH CORTICOSTEROIDS VERSUS ARTHROCENTESIS WITH HYALURONIC ACID IN THE MANAGEMENT OF TEMPOROMANDIBULAR JOINT OSTEOARTHRITIS
}

\author{
Salah El-Abbasy *
}

\begin{abstract}
Aim: to evaluate the clinical results, radiographic results and levels of inflammatory mediators following arthrocentesis with intra-articular injection of steroids versus arthrocentesis with intraarticular injection of hyaluronic acid in temporomandibular joint osteoarthritis.
\end{abstract}

Materials and Methods: Ten patients suffering from Osteoarthritis with pain and crepitation during jaw movements together with radiographic evidence of bony erosions were included in the study. The patients were divided into two groups, five patients in group A were treated with arthrocentesis followed by intra-articular injection of corticosteroid and five patients in group B were treated with arthrocentesis followed by intra-articular injection of Hyaluronic acid.

Results: Patients in both groups showed significant reduction in pain and increase in maximum mouth opening; however, arthrocentesis with intra-articular injection of HA showed better clinical results compared to arthrocentesis with intra-articular injection of corticosteroids. Less inflammatory mediators were found in the Corticosteroid group patients compared to HA group patients. No evidence of new bone formation was seen in both groups after 4 months postoperatively.

Conclusion: Better clinical results were achieved after Arthrocentesis followed by intraarticular injection of hyalurionic acid when compared to arthrocentesis with intra-articular injection of corticosteroids. Less inflammatory mediators were associated with arthrocentesis with corticosteroids injection compared to arthrocentesis with HA injection. Long term follow up is required to be able to assess new bone formation following arthrocentesis with intra-articular injection of HA or corticosteroid.

KEYWORDS: Osteoarthritis, Hyaluronic acid, Arthrocentesis, Corticosteroids

* Lecturer of Oral and Maxillofacial Surgery, Oral and Maxillofacial Surgery Department, Faculty Dentistry, Cairo University. 


\section{INTRODUCTION}

Osteoarthritis (OA) is degenerative diseases that can result in total destruction of joint structures. ${ }^{1}$ Diagnosis of OA is carried out throw the criteria described by Dworkin and LeResche ${ }^{2}$ based on the bony changes of the articulating surfaces of the joint, crepitation on jaw movements and pain on mouth opening.

Many conservative approaches were carried out for the management of temporomandibular joint (TMJ) OA including arthrocentesis, injection of hyaluronic acid(HA), physical exercises, injection of steroids and acupuncture. ${ }^{3}$

Arthrocentesis is a simple, minimally invasive technique that breaks up the joint adhesions and wash away the inflammatory mediators and necrotic tissues from the joint. ${ }^{4}$ A study by Gulen et al ${ }^{5}$ resulted in significant decrease of interleukins and tumour necrosis factor alpha in the synovial fluid following arthrocentesis.

Hyaluronic acid can act as a lubricant and provides nutrition to the meniscus and showed excellent results in treating large joints. ${ }^{6}$ The combination between arthrocentesis and HA injection was found to have much better results when compared to arthrocentesis alone in the treatment of osteoarthritis in horses. ${ }^{7}$

Corticosteroids are anti-inflammatory drugs that have been used for therapeutic and diagnostic purposes. ${ }^{8}$ Many studies examined the intraarticular use of corticosteroids and final positive results were achieved..$^{910}$ The combination between arthrocentesis and corticosteroids injection resulted in better function and less pain with mandibular movements. ${ }^{11}$

A study was performed to compare the results between the effects of HA injection and corticosteroid injection without arthrocentesis for patients suffering from OA. Both drugs resulted in significant reduction of pain but comparing between the side effects of the two drugs, the HA was found to be superior over the corticosteroids. ${ }^{?}$

\section{MATERIALS AND METHODS}

Ten patients suffering from OA of the TMJ with an age range between 35 and 65 years and a mean age of $49.3 \pm 5.4$ years were selected from the Department of Oral and Maxillofacial Surgery, Faculty of Dentistry, Cairo University. Inclusion criteria included cases with OA associated with pain and crepitation during jaw movements and a radiographic evidence of OA in $\mathrm{CT}$ scans showing bone erosions, or flattening in the condyle and/or the articulating fossa.

The patients were divided into 2 groups; group A including 5 patients performed arthrocentesis followed by intra-articular injection of corticosteroid and group B including 5 patients performed arthrocentesis followed by intra-articular injection of HA. Any patients with severe systemic diseases, autoimmune disease, coagulation disorders, pregnancy or lactation and those patients with previous TMJ injections were excluded from the study. All the cases were performed under local anaethesia.

Conservative management was carried out for all the patients in terms of non steroidal antiinflammatory drugs, physiotherapy, patient education and splints before they were included in the study.

A straight line was drawn on the patients face from the outer canthus of the eye till the middle of the tragus of the ear. The posterior point of entrance was located along this line, $1 \mathrm{~cm}$ anteriorly to the middle of the tragus and $2 \mathrm{~mm}$ below the line while the anterior entrance point was located $2 \mathrm{~cm}$ anteriorly to the middle of the tragus of the ear and $1 \mathrm{~cm}$ below the canthotragal line.

Arthrocentesis procedure was then started by injecting Articaine 4\% with epinephrine 1:100000 at the entrance points. Patients were asked to open their mouth widely and protrude their mandible. An 18-gauge needle was inserted into the posterior entrance point in the superior joint space. A $5 \mathrm{ml}$ Ringer's lactate solution was then injected into the joint prior to the insertion of the second needle to 
distend the superior joint space and release any adhesions. The second needle was then inserted through the anterior entrance point to allow the lactated Ringer's solution to flow freely through the superior joint space.

Ringer's lactate solution (300-400 ml) was injected simultaneously from both needles (Fig.1) and allowed to pass through the superior joint space and during the process of injection, the patients were asked to move their mandible in various directions including opening, closing, protrusive and lateral excursions to facilitate the lysis of any adhesions.

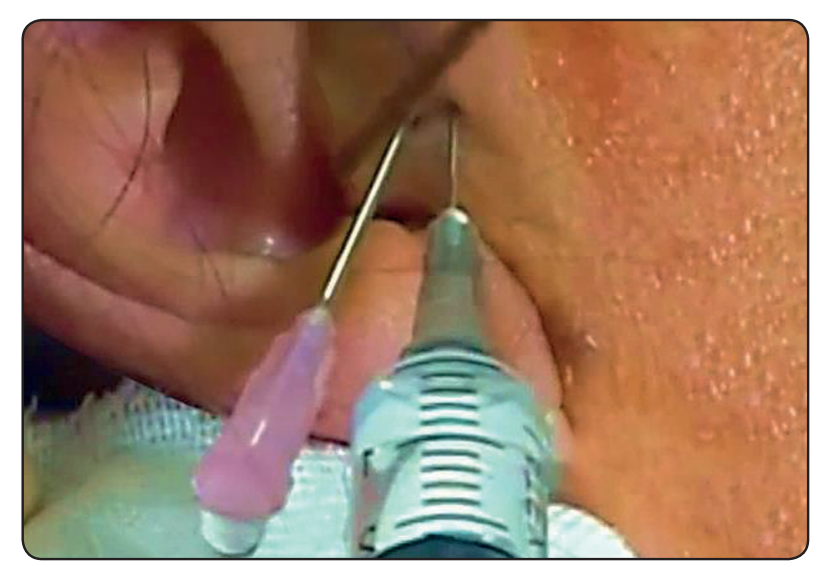

Fig. (1) Free flowing of the lactated Ringer's solution through the superior joint space.

This procedure was performed for all the patients in both groups. The anterior needle was then removed followed by intra-articular injection of $1 \mathrm{ml}$ of betamethasone (Betafos $7 \mathrm{mg} / 2 \mathrm{ml}$ ampoule, Amoun Pharmaceuticals, Egypt) in group A patients and $1 \mathrm{ml}$ of HA (Curavisk $20 \mathrm{mg} / 2 \mathrm{ml}$ syringe, Curasan, Germany) in group B patients, then the posterior needle was removed and a pressure pack was placed over the injection points.

A sample from the joint fluid was collected preoperatively and after 4 months postoperatively through the puncture of an 18-gauge needle into the superior joint space followed by simultaneous injection and aspiration of 1 $\mathrm{cc}$ of saline solution for five times and finally transferring the sample to the lab to examine the levels of tumor necrosis factor alpha (TNF- $\alpha$ ) and interleukin (IL-6).

Follow up was carried out immediate postoperatively and after 1 week, 2 weeks, 4 weeks, 2 months and 4 months postoperatively in terms of:

- Pain with various mandibular movements utilizing the Visual analogue scale (VAS).

- $\quad$ Maximum mouth opening (MMO) by measuring the distance between the upper central and lower central incisors in millimeters.

However preoperative and 4 months postoperative records were obtained in terms of:

- Clicking/crepitus in the joint.

- A sample from the joint fluid was taken to examine the levels of tumor necrosis factor alpha (TNF- $\alpha$ ) and interleukin (IL-6).

- Radiological assessment of the joint with CT scans.

\section{RESULTS}

Ten selected patients (30\% males and $70 \%$ females) were divided randomly into two groups. Five patients ( 2 males and 3 females) were included in group A and five patients (1 male and 4 females) were included in group B. Arthrocentesis followed by Corticosteroid injection was performed for group A patients, while arthrocentesis followed by injection of HA was performed for group B patients.

According to the VAS of pain, significant decrease in pain was observed in the two groups at the end of the follow up period; however pain reduction was more significant in group B patients when compared to group A (Table 1).

During different follow up periods, patients were classified according to the severity of pain where by the end of the follow up period all the patients in both groups were presented with no pain or mild pain (Table 2).

A sample from Joint fluid was collected preoperatively and after 4 months postoperatively and the levels of interleukin 6 (IL-6) and tumour necrosis factor $\alpha$ (TNF- $\alpha)$ was measured. 
A significant decrease in the level of IL-6 and TNF- $\alpha$ in joint fluid was observed after 4 months postoperatively in both groups; however more decrease was noticed in group A patients compared to group B (Table 4, 5).

Preoperatively, all the patients in both groups were suffering from clicking and crepitus during different jaw movements, and at the end of the follow up period, clicking sound significantly decreased but there was no significant change in crepitation sound.

CT scans preoperatively and after 4 months postoperatively showed slight difference concerning bone remodeling but no evidence of new bone formation was observed in both groups (Fig. 2,3).

TABLE (1) Comparison of the mean pain scores between group A and B during different follow up periods.

\begin{tabular}{|c|c|c|c|}
\hline \multirow{2}{*}{ Time period } & \multicolumn{2}{|c}{ Mean SD } & \multirow{2}{*}{ P } \\
\cline { 2 - 4 } & GROUP A & GROUP B & 0.78 \\
\hline Pre-op & 5.41 .14 & 5.61 .14 & 0.24 \\
\hline Immediate post-op & 5.61 .14 & 4.80 .83 & 0.07 \\
\hline Week 1 & 5.01 .22 & 3.80 .44 & 0.03 \\
\hline Week 2 & 4.20 .83 & 3.00 .70 & 0.02 \\
\hline Week 4 & 3.80 .83 & 2.60 .54 & 0.007 \\
\hline 2 Months & 3.40 .54 & 2.00 .70 & 0.01 \\
\hline
\end{tabular}

$* P<0.05$ Significant

TABLE (2) Severity of pain during different follow up periods.

\begin{tabular}{|c|c|c|c|c|c|c|c|}
\hline \multirow{2}{*}{$\begin{array}{c}\text { Pain } \\
\text { (VAS) }\end{array}$} & \multicolumn{7}{|c|}{ Number of Patients } \\
\hline & Preop & Postop & Week 1 & Week 2 & Week 4 & 2 Month & 4 Month \\
\hline \multicolumn{8}{|l|}{ Group A } \\
\hline No to mild pain & 0 & 1 & 1 & 1 & 4 & 5 & 5 \\
\hline Moderate pain & 4 & 4 & 4 & 4 & 1 & 0 & 0 \\
\hline Severe pain & 1 & 0 & 0 & 0 & 0 & 0 & 0 \\
\hline Worst Pain & 0 & 0 & 0 & 0 & 0 & 0 & 0 \\
\hline \multicolumn{8}{|l|}{ Group B } \\
\hline No to mild pain & 0 & 0 & 0 & 0 & 3 & 5 & 5 \\
\hline Moderate pain & 3 & 4 & 5 & 5 & 2 & 0 & 0 \\
\hline Severe pain & 2 & 1 & 0 & 0 & 0 & 0 & 0 \\
\hline Worst Pain & 0 & 0 & 0 & 0 & 0 & 0 & 0 \\
\hline
\end{tabular}

TABLE (3) Comparison of MMO during follow up periods between both groups.

\begin{tabular}{|c|c|c|c|}
\hline \multirow{2}{*}{ Time period } & \multicolumn{2}{|c|}{ Mean SD } & P \\
\cline { 2 - 4 } & Group A & Group B & 0.11 \\
\hline Pre-operative & 35.32 .08 & 34.91 .62 & 0.36 \\
\hline Immediate postop & 37.42 .32 & 38.31 .57 & 0.03 \\
\hline Week 1 & 37.52 .72 & 39.61 .58 & 0.05 \\
\hline Week 2 & 39.72 .42 & 41.20 .95 & 0.004 \\
\hline Week 4 & 41.10 .65 & 42.30 .96 & 0.008 \\
\hline 2 Months & 42.12 .2 & 44.61 .59 & 0.004 \\
\hline
\end{tabular}


TABLE (4) Comparing interleukin 6 levels between the 2 groups.

\begin{tabular}{|c|c|c|c|}
\hline \multirow{2}{*}{} & \multicolumn{2}{|c|}{ MeanSD of IL-6 levels } & \multirow{2}{*}{ P } \\
\cline { 2 - 3 } & Group A & Group B & \\
\hline Preoperative & 36.155 .93 & 36.827 .35 & 0.82 \\
\hline 4 months & 25.1211 .51 & 27.747 .35 & 0.64 \\
\hline P & 0.0001 & 0.0001 & \\
\hline
\end{tabular}

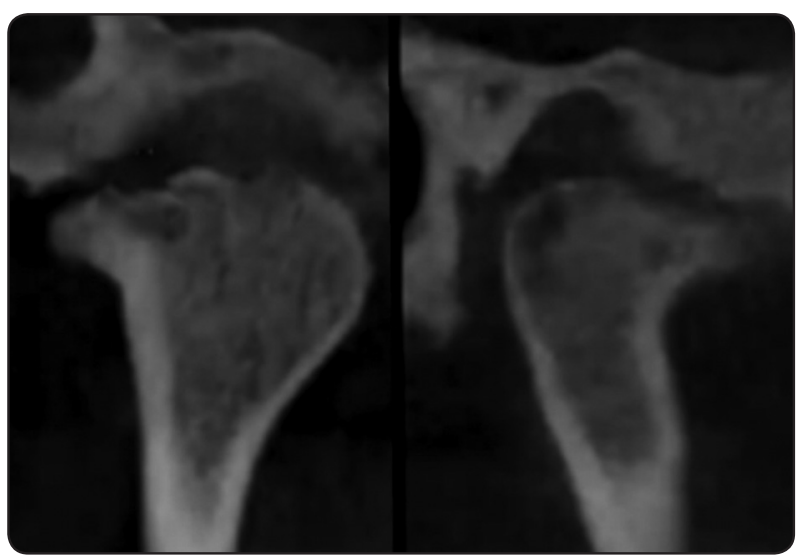

Fig. (2) Preoperative CT scan showing bony erosions in the condyle and eminence.

\section{DISCUSSION}

In this study, all the patients were suffering from moderate to severe pain but there was a significant decrease in pain along the follow up periods in both groups, however more reduction in pain was noticed in HA group compared to corticosteroid group. This agrees with the studies of Alpalsan and Alpalsan ${ }^{12}$, Cascone et al ${ }^{13}$ and Manfredini et al ${ }^{14}$ who reported siginificant decrease in pain scores after performing arthrocentesis with HA injection and attributed this results to the the effects of fluid pressure from arthrocentesis, washing away of the inflammatory mediators and the lubrication action of the HA. Our result also agrees with Koop et al ${ }^{9}$ reporting significant decrease in TMJ pain following injection of corticosteroids and hyaluronic acid.
TABLE (5) Comparing TNF- $\alpha$ levels between the 2 groups.

\begin{tabular}{|c|c|c|c|}
\hline & \multicolumn{2}{|c|}{ MeanSD of TNF $\alpha$ levels } & P \\
\hline & Group A & Group B & \\
\hline Preoperative & 22.458 .59 & 31.659 .11 & 0.03 \\
\hline 4 months & 14.474 .64 & 27.678 .12 & 0.001 \\
\hline P & 0.0001 & 0.0001 & \\
\hline
\end{tabular}

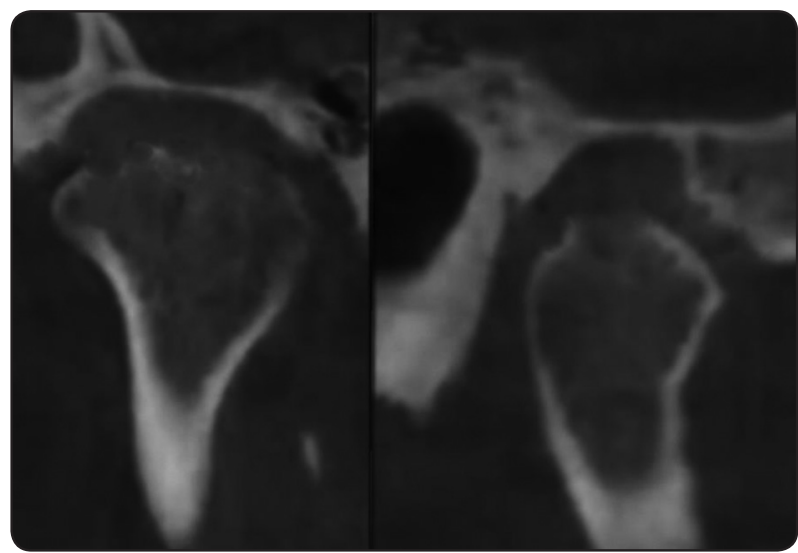

Fig. (3) CT scan showing no evidence of new bone formation after 4 months postoperatively.

In our study, the maximal interincisal distance was found to increase during different follow up periods in both groups, however there was a more significant increase in HA group patients when compared to the corticosteroid group. This is consistent with the study of Manfredini et al ${ }^{14}$ who reported an increase in maximum mouth opening following arthrocentesis with HA injection. Our results also agree with Yeung et al ${ }^{15}$ and Dolwick ${ }^{16}$ who reported an increase of MMO following intra articular injection of HA. The authors attributed these results to the lubricant and analgesic properties of HA, decreases in the frictional coefficient in the joint and the release of adhesions resulting from the fluid pressure. 
In the current study, the patients in both groups were presented with no or mild pain with lateral and protrusive movements at the end of the follow up period. This supports the findings of Alpalsan and Alpalsan ${ }^{12}$ and Neo et al ${ }^{17}$ who reported that lateral movements were improved during different follow up periods following arthrocentesis and they attributed that to the breaking up of joint adhesions, increase in lubrication by HA and suppressing the inflamatory mediators by corticosteroids.

In the present study, clicking sound significantly decreased in all the patients in both groups, however crepitation sounds remained unchanged after 4 months postoperatively. This coincides with the findings of Yeung et al ${ }^{15}$ but disagrees with the findings of Alpalsan and Alpalsan ${ }^{12}$ who reported that there was no significant decrease of clicking sounds following arthrocentesis.

In this study, the levels of IL-6 and TNF- $\alpha$ decreased significantly in both groups after 4 months postoperatively, however more decrease in levels was found in the corticosteroid group when compared to HA group. This is consistent with the studies performed by Takahashi et al ${ }^{18}$ and Gulen et al ${ }^{5}$ who reported that inflammatory mediators are normally absent in healthy joints and there was a significant decrease in inflammatory mediators following arthrocentesis for unhealthy joints. Our results was also found to be in agreement with Sezgin et al ${ }^{19}$ reporting that IL-6 levels decreased significantly after HA injection in knee joints. Comert ${ }^{20}$ reported that corticosteroids affects the synovial tissues by suppressing the inflammatory molecules and passing through the cellular membrane and and the binding receptors located in the cytoplasm where these activated receptors eliminate the expression of pro inflammatory cytokines and inflammatory mediators.

In our study, CT scans showed slight bone remodeling but new bone formation wasn't evident after 4 month postoperatively in both groups. This meets the findings of $\mathrm{Li}$ et al ${ }^{21}$ reporting that at least 9 months is required in order to observe new bone formation following intra-articular injection of HA.

\section{CONCLUSION}

Better clinical results were achieved after Arthrocentesis followed by intra-articular injection of hyalurionic acid when compared to arthrocentesis with intra-articular injection of corticosteroids. Less inflammatory mediators were associated with arthrocentesis with corticosteroids injection compared to arthrocentesis with HA injection. Long term follow up is required to be able to assess new bone formation following arthrocentesis with intraarticular injection of HA or corticosteroid.

\section{REFERENCES}

1. Schiffman E, Ohrbach R, Truelove E, Look J, Anderson G, Goulet JP: Diagnostic criteria for temporomandibular disorders (DC/TMD) for clinical and research applications: Recommendations of the international RDC/TMD consortium network $*$ and orofacial pain special interest group $\dagger \mathrm{J}$ Oral Facial Pain Headache; 28:6-27, 2014.

2. Dworkin SF, LeResche L. :Research diagnostic criteria for temporomandibular disorders:Review, criteria, examinations and specifications, critique. J Craniomandib Disord; 6:301-55, 1992

3. Gadek A, Miskowiec K, Wordliczek J, Liszka H. : Effectiveness and safety of intra-articular use of hyaluronic acid (Suplasyn) in the treatment of knee osteoarthritis. Przegl Lek; 68:307-10, 2011.

4. Emshoff R, Puffer P, Strobl H, Gassner R.: Effect of temporomandibular joint arthrocentesis on synovial fluid mediator level of tumor necrosis factor-alpha: implications for treatment outcome. Int J Oral Maxillofac Surg; 29:176-82, 2000.

5. Gulen H, Ataoglu H, Haliloglu S, Isik K. :Proinflammatory cytokines in temporomandibular joint synovial fluid before and after arthrocentesis. Oral Surg Oral Med Oral Pathol Oral Radiol Endod; 107:e1-4, 2009.

6. Khalaj N, Abu Osman NA, Mokhtar AH, George J, Abas WA. :Effect of intra-articular hyaluronic injection on postural stability and risk of fall in patients with bilateral knee osteoarthritis. ScientificWorldJournal ; 8:151-184, 2014. 
7. Brusie RW, Sullins KE, White NA, 2nd, Coffin PC, Parker GA, Anver MR.: Evaluation of sodium hyaluronate therapy in induced septic arthritis in the horse. Equine Vet $\mathrm{J}$ Suppl; 11:18-23, 1992.

8. Yura S, Totsuka Y, Yoshikawa T, Inoue N. :Can arthrocentesis release intracapsular adhesions? Arthroscopic findings before and after irrigation under sufficient hydraulic pressure. J Oral Maxillofac Surg; 61: 1253-1256, 2003.

9. Koop S, Carlsson GE, Haraldson T, Wenneberg B. :The short term effect of intra-articular injection of sodium hyaluronate and corticosteroid on temporomandibular joint pain and dysfunction. J Oral Maxillofac Surg ; 43:429435, 1985.

10. Koop S, Carlsson GE, Haraldson T, Wenneberg B. :The long term effect of intra-articular injection of sodium hyaluronate and corticosteroid on temporomandibular joint arthritis. J Oral Maxillofac Surg; 43:929-935, 1987.

11. Nitzan DW, Dolwick MF, Heft MW. :Arthroscopic lavage and lysis of the TMJ: a change in perspective. J Oral Maxillofac Surg; 48:798-801, 1990.

12. Alpaslan GH, Alpaslan C. :Efficacy of temporomandibular joint arthrocentesis with and without injection of sodium hyaluronate in treatment of internal derangements. J Oral Maxillofac Surg ; 59:613-8, 2001.

13. Cascone P, Fonzi Dagger L, Aboh IV. :Hyaluronic acid's biomechanical stabilization function in the temporomandibular joint. J Craniofac Surg;13:751-4, 2002.

14. Manfredini D, Bonnini S, Arboretti R, Guarda-Nardini L. :Temporomandibular joint osteoarthritis: An open label trial of 76 patients treated with arthrocentesis plus hyaluronic acid injections. Int J Oral Maxillofac Surg; 38:827-34, 2009.
15. Yeung RW, Chow RL, Samman N, Chiu K. :Short-term therapeutic outcome of intra-articular high molecular weight hyaluronic acid injection for nonreducing disc displacement of the temporomandibular joint. Oral Surg Oral Med Oral Pathol Oral Radiol Endod;102:453-61, 2006.

16. Dolwick MF. :The role of temporomandibular joint surgery in the treatment of patients with internal derangement. Oral Surg Oral Med Oral Pathol Oral Radiol Endod; 83:150-5, 1997.

17. Neo H, Ishimaru JI, Kurita K, Goss AN. : The effect of hyaluronic acid on experimental temporomandibular joint osteoarthrosis in the sheep. J Oral Maxillofac Surg; 55:1114-9, 1997.

18. Takahashi T, Kondoh T, Fukuda M, Yamazaki Y, Toyosaki T, Suzuki R.: Proinflammatory cytokines detectable in synovial fluids from patients with temporomandibular disorders. Oral Surg Oral Med Oral Pathol Oral Radiol Endod; 85:135-41, 1998.

19. Sezgin M, Demirel AC, Karaca C, Ortancil O, Ulkar GB, Kanik A: Does hyaluronan affect inflammatory cytokines in knee osteoarthritis? Rheumatol Int; 25:264-9, 2005.

20. Comert Kilic S. :Does Injection of Corticosteroid After Arthrocentesis Improve Outcomes of Temporomandibular Joint Osteoarthritis? A Randomized Clinical Trial. J Oral Maxillofac Surg; 74:2151-8, 2016.

21. Li C, Long X, Deng M, Li J, Cai H, Meng Q. :Osteoarthritic changes after superior and inferior joint space injection of hyaluronic acid for the treatment of temporomandibular joint osteoarthritis with anterior disc displacement without reduction: A cone-beam computed tomographic evaluation. J Oral Maxillofac Surg; 73:232-44, 2015. 